\title{
Endovascular repair of a thoracoabdominal aortic aneurysm using a physician-modified four-vessel fenestrated endograft
}

\author{
Dean J. Arnaoutakis ${ }^{1}$, Micaela R. Cuneo ${ }^{2}$, George J. Arnaoutakis ${ }^{2}$ \\ ${ }^{1}$ Division of Vascular Surgery, University of South Florida, Tampa, FL, USA; ${ }^{2}$ Division of Cardiovascular Surgery, University of Florida, Gainesville, \\ FL, USA \\ Correspondence to: George J. Arnaoutakis, MD. Associate Professor, Division of Cardiovascular Surgery, UF Health, University of Florida College of \\ Medicine, Gainesville, FL 32607, USA. Email: George.arnaoutakis@surgery.ufl.edu.
}

Submitted Jan 05, 2022. Accepted for publication Jan 17, 2022.

doi: 10.21037/acs-2022-taes-27

View this article at: https://dx.doi.org/10.21037/acs-2022-taes-27

\section{Clinical vignette}

A 68-year-old female presented with a surgical history of aortic arch debranching with concomitant antegrade thoracic endovascular aortic repair (TEVAR), performed as a frozen elephant trunk. Past medical history includes chronic obstructive pulmonary disease (COPD) with active tobacco use, hypertension, hyperlipidemia, and type 2 diabetes mellitus. Two years after her TEVAR, she had persistent $6 \mathrm{~cm}$ extent III thoracoabdominal aortic aneurysm (TAAA). She denied any abdominal, back, or chest pain. Computed tomography angiography (CTA) showed an aneurysm beginning at the distal end of the previously placed TEVAR. She was deemed a poor open operative candidate due to her age and COPD with tobacco use and was offered a total endovascular repair. To mitigate spinal cord ischemia, staged repair was recommended. The first stage consisted of a TEVAR to the level of the celiac artery, and the second stage involved deployment of a surgeon-modified 4-vessel fenestrated endograft one month later. A spinal drain was placed preoperatively given the length of aortic coverage.

\section{Surgical techniques}

\section{Preparation}

After vascular access was obtained and anesthesia induced, the patient was positioned supine with the arm elevated above the head and prepped and draped in standard sterile fashion.

\section{Operation}

\section{Stage I}

An 8-F sheath was inserted into the right common femoral artery (RCFA) after deploying two ProGlide devices (Abbott, Abbott Park, IL, USA). A pigtail catheter was inserted through a 5-F sheath in the left common femoral artery (LCFA) and positioned in the descending thoracic aorta and angiography performed to mark the celiac artery origin. After dilating the RCFA, a Cook Alpha 36-32/209 thoracic device (Cook Medical, Bloomington, IN, USA) was inserted with 5-6 cm of overlap with the previous TEVAR (elephant trunk) device and distally positioned $5 \mathrm{~cm}$ proximal to the celiac artery. After deployment, a Cook Alpha 32-109 mm device was introduced and deployed such that it landed $1-2 \mathrm{~cm}$ proximal to the celiac artery with at least $6 \mathrm{~cm}$ of overlap with the more proximal device. Completion angiography showed no evidence of type Ia, II, or III endoleak. As expected, there was a type Ib endoleak given the distal end of the endograft was positioned in the aneurysm. The ProGlide devices sealed the RCFA, and manual pressure was applied on the LCFA. The patient was extubated and transferred to the surgical intensive care unit (SICU).

\section{Stage II}

Approximately one month later, the patient returned for the second stage. A lumbar drain was placed preoperatively. On a sterile back-table, a Cook Alpha 32/201 mm device (Cook Medical, Bloomington, IN, USA) was unsheathed and 
removed from the delivery system. Four fenestrations were created with eye-cautery in the appropriate locations based on centerline reconstruction of the preoperative CTA. The fenestrations were reinforced with Nester coils (Cook Medical, Bloomington, IN, USA) and $6 \mathrm{~mm}$ thin-walled PTFE using 6-0 Gore-Tex suture in a running fashion (W. L. Gore Inc., Flagstaff, AZ, USA). The graft was then reattached to the delivery system and partially constrained using multiple 4-0 chromic sutures.

After gaining femoral access, limited angiography was performed of the visceral-renal segment of the aorta to refine our CT fusion marks delineating the origins of the aortic-branch vessels. The 4-vessel fenestrated device was then inserted through the RCFA and deployed, aligning the fenestrations with the CT fusion marks. Graft constraint permitted minor longitudinal and rotational adjustment of the device. Through an 18-F sheath in the LCFA, a wire was advanced into the distal opening of the fenestrated device. The $18-\mathrm{F}$ sheath was then advanced and sequential target vessel cannulation ensued using a 7-F Aptus sheath (Medtronic, Dublin, Ireland), a support catheter and Glidewire. After selecting the fenestration and target vessel, angiography was performed to confirm location and a Rosen wire advanced into the vessel.

This sequence occurred for each renal artery, superior mesenteric artery and celiac artery. With fenestration cannulation complete, the endograft was released and a 16-F sheath inserted. A semi-compliant balloon was introduced to treat the proximal overlap between components and to disrupt the chromic sutures constraining the endograft. Next, appropriately sized iCast stents (Atrium Medical, Merrimack, NH, USA) were sequentially inserted as bridging stents and deployed into each target vessel over the Rosen wires. The origin of each iCast stent was flared with a balloon to prevent type IIIc endoleak. In this case, distal seal was obtained in the terminal aorta, and no additional endografts were needed. Completion imaging showed excellent flow into visceral and renal arteries with no type I, II, or III endoleak. The patient was extubated and transferred to the SICU.

\section{Postoperative recovery}

The patient recovered uneventfully and the spinal drain was clamped postoperative day 1 for both procedures. Mean arterial pressures were intentionally kept elevated. She remained free of neurologic compromise, and her spinal drain was removed the next day. She was discharged postoperative day two, taking aspirin $81 \mathrm{mg}$ and clopidogrel $75 \mathrm{mg}$ daily. Our standard imaging surveillance involves postoperative CTA at 1-month, 6-month, and annually thereafter. Her 8-month scan showed stable operative changes with patency along the graft and stents and no evidence of endoleak or aneurysm expansion.

\section{Comments}

Total endovascular repair of TAAA's has emerged as a safe and effective treatment option particularly useful in patients with multiple comorbid conditions (1). However, similar to traditional open surgery, spinal cord ischemia remains of great concern and albeit uncommon, is devastating and portends a poor 1-year survival (2). When extensive aortic coverage is involved, staging of procedures is critical to mitigate spinal cord risk.

Most elderly patients with combined arch and TAAA pathology undergo a hybrid repair as described (arch debranching with FET followed by fenestrated/branched repair of the thoracoabdominal aorta). However, as aortic arch endograft design and access continues to advance, limited series have demonstrated technical success and safety of a complete endovascular repair in these patients (3).

While technology continues to evolve, currently there are no commercially available devices to treat thoracoabdominal or aortic arch pathology. Patients who are not open candidates must either seek a center with access to a customized aortic program through physiciansponsored investigational device exemption or a clinical device research trial. Accordingly, acute pathologies are not easily treatable unless patients are in close proximity to such centers. As technology in this arena becomes more mature, an increasing number of patients will be able to successfully undergo endovascular treatment.

\section{Acknowledgments}

Funding: None.

\section{Footnote}

Conflicts of Interest: The authors have no conflicts of interest to declare.

Open Access Statement: This is an Open Access article distributed in accordance with the Creative Commons Attribution-NonCommercial-NoDerivs 4.0 International 
License (CC BY-NC-ND 4.0), which permits the noncommercial replication and distribution of the article with the strict proviso that no changes or edits are made and the original work is properly cited (including links to both the formal publication through the relevant DOI and the license). See: https://creativecommons.org/licenses/by-nc-nd/4.0/.

\section{References}

1. Arnaoutakis DJ, Scali ST, Beck AW, et al. Comparative

Cite this article as: Arnaoutakis DJ, Cuneo MR, Arnaoutakis GJ. Endovascular repair of a thoracoabdominal aortic aneurysm using a physician-modified four-vessel fenestrated endograft. Ann Cardiothorac Surg 2022;11(1):65-67. doi: 10.21037/acs2022-taes-27 outcomes of open, hybrid, and fenestrated branched endovascular repair of extent II and III thoracoabdominal aortic aneurysms. J Vasc Surg 2020;71:1503-14.

2. DeSart K, Scali ST, Feezor RJ, et al. Fate of patients with spinal cord ischemia complicating thoracic endovascular aortic repair. J Vasc Surg 2013;58:635-42.e2.

3. Tsilimparis N, Haulon S, Spanos K, et al. Combined fenestrated-branched endovascular repair of the aortic arch and the thoracoabdominal aorta. J Vasc Surg 2020;71:1825-33. 